Volume 132, Number 4, Pages 1133-1142

S 0002-9939(03)07152-1

Article electronically published on June 23, 2003

\title{
APPROXIMATION IN REFLEXIVE BANACH SPACES AND APPLICATIONS TO THE INVARIANT SUBSPACE PROBLEM
}

\author{
ISABELLE CHALENDAR, JONATHAN R. PARTINGTON, AND MARTIN SMITH
}

(Communicated by N. Tomczak-Jaegermann)

\begin{abstract}
We formulate a general approximation problem involving reflexive and smooth Banach spaces, and give its explicit solution. Two applications are presented - the first is to the Bounded Completion Problem involving approximation of Hardy class functions, while the second involves the construction of minimal vectors and hyperinvariant subspaces of linear operators, generalizing the Hilbert space technique of Ansari and Enflo.
\end{abstract}

\section{INTRODUCTION}

The construction of minimal vectors, corresponding to an operator on a Hilbert space, was introduced by Ansari and Enflo [3], who used it to give simpler proofs of the existence of invariant subspaces for certain classes of operators, including compact operators and normal operators. The minimal vectors are given as the solution of certain constrained approximation problems, and in [9] a more general such problem was formulated and solved, which has other applications, notably in the theories of systems identification, signal processing and inverse problems (see [8] for a survey of this area).

It is possible to define minimal vectors in a more general Banach space context, although the associated approximation problem is rather more difficult to resolve. Androulakis 2] generalized the techniques of Ansari and Enflo to produce a new sufficient condition for the existence of hyperinvariant subspaces of certain operators on super-reflexive Banach spaces. Troitsky [18] extended the method of Androulakis and obtained a similar result for arbitrary Banach spaces. In this paper we extend the methods of 3 in a different way by solving an approximation problem in the class of reflexive Banach spaces: it does not seem likely that such a construction is valid in the class of nonreflexive spaces.

We shall employ the following notation.

For $\mathcal{X}$ a complex Banach space, $\mathcal{L}(\mathcal{X})$ denotes the algebra of all bounded linear operators on $\mathcal{X}$. Suppose that $T \in \mathcal{L}(\mathcal{X})$. We denote by $\sigma(T)$ the spectrum of $T$ and we say that $T$ is quasinilpotent if its spectrum is equal to $\{0\}$. Equivalently, $T$ is quasinilpotent if and only if $\lim _{n \rightarrow \infty}\left\|T^{n}\right\|^{1 / n}=0$. A nontrivial hyperinvariant

Received by the editors October 7, 2002 and, in revised form, December 17, 2002.

2000 Mathematics Subject Classification. Primary 41A29, 47A15, 46B20, 46E15.

Key words and phrases. Constrained approximation, smoothness, invariant subspaces, Hardy spaces, extremal problems. 
subspace of $T$ is a closed subspace $\mathcal{M}$ such that $\{0\} \neq \mathcal{M} \neq \mathcal{X}$ and $A \mathcal{M} \subseteq \mathcal{M}$ for all $A \in\{T\}^{\prime}:=\{A \in \mathcal{L}(\mathcal{X}): A T=T A\}$.

A Banach space $\mathcal{X}$ is said to be smooth if for every vector $x \in \mathcal{X} \backslash\{0\}$ there is a unique linear functional $\nu_{\mathcal{X}}(x)$ in $\mathcal{X}^{*}$ such that $\left\|\nu_{\mathcal{X}}(x)\right\|=1$ and $\nu_{\mathcal{X}}(x) x=\|x\|$. When there is no ambiguity, we shall write $\nu(x)$ for $\nu_{\mathcal{X}}(x)$. Furthermore, $\mathcal{X}$ is said to be strictly convex if, whenever $x$ and $y$ are linearly independent vectors in $\mathcal{X}$, we have $\|x+y\|<\|x\|+\|y\|$.

We write $\mathbb{T}$ for the unit circle $\{z \in \mathbb{C}:|z|=1\}$, and $L^{p}(\mathbb{T})$ and $H^{p}(\mathbb{T})$ for the classical Lebesgue and Hardy spaces.

The plan of the paper is the following. First, we formulate a general extremal problem involving a reflexive Banach space $\mathcal{X}$ and smooth Banach spaces $\mathcal{Y}$ and $\mathcal{Z}$; we give an explicit solution, which is unique when in addition $\mathcal{X}$ is strictly convex.

Then, in Section 3 we give two kinds of applications. The first is the resolution of the so-called Bounded Completion Problem (BCP) in a reflexive, smooth and strictly convex Banach space. The second concerns the Invariant Subspace Problem. Indeed, following the Hilbert space techniques introduced by Ansari and Enflo, and generalizing them to reflexive Banach spaces, we obtain sufficient conditions for the existence of hyperinvariant subspaces for some operators that are not necessarily compact nor quasinilpotent, as shown by explicit examples.

\section{A general extremal problem}

In order to formulate the extremal problem of which we shall later see applications, let $\mathcal{X}, \mathcal{Y}$ and $\mathcal{Z}$ be complex Banach spaces, such that $\mathcal{X}$ is reflexive and $\mathcal{Y}$ and $\mathcal{Z}$ are smooth, and let $A: \mathcal{X} \rightarrow \mathcal{Y}$ and $B: \mathcal{X} \rightarrow \mathcal{Z}$ be bounded linear operators, such that $A$ and $B$ are coprime, in the sense that there exists a constant $\eta>0$ such that

$$
\|A x\|+\|B x\| \geq \eta\|x\| \quad \text { for all } x \in \mathcal{X} .
$$

Let $t_{0} \in \mathcal{Z}$ and $t_{1} \in \mathcal{Y}$ be fixed vectors. For $\varepsilon>0$ we consider the closed convex set

$$
\mathcal{C}_{t_{0}}^{\varepsilon}=\left\{x \in \mathcal{X}:\left\|B x-t_{0}\right\| \leq \varepsilon\right\},
$$

and we suppose that $\varepsilon$ is chosen so large that $\mathcal{C}_{t_{0}}^{\varepsilon}$ has nonempty interior. We suppose also that $A x \neq t_{1}$ for all $x \in \mathcal{C}_{t_{0}}^{\varepsilon}$ (to eliminate a degenerate case). In this situation the associated extremal problem is to find $x_{0} \in \mathcal{C}_{t_{0}}^{\varepsilon}$ such that

$$
\left\|A x_{0}-t_{1}\right\|=\inf \left\{\left\|A x-t_{1}\right\|: x \in \mathcal{C}_{t_{0}}^{\varepsilon}\right\}=\beta, \quad \text { say. }
$$

In view of the applications that we shall discuss, we also assume that $A$ has dense range.

The above extremal problem provides a simultaneous generalization of the extremal problems discussed in 9] and [17. These in turn provided extensions in different directions of a Hilbert space problem analysed in [12], where $\mathcal{X}$ was a closed subspace of $\mathcal{Y} \oplus \mathcal{Z}$, and $A$ and $B$ were the restrictions to $\mathcal{X}$ of the orthogonal projections onto $\mathcal{Y}$ and $\mathcal{Z}$, respectively. A simple example of this is the case when $\mathcal{X}$ is the Hardy space $H^{2}(\mathbb{T}), \mathcal{Y}=L^{2}(I)$ and $\mathcal{Z}=L^{2}(J)$, where $I$ and $J$ are complementary subsets of the unit circle, each with strictly positive measure. This version of the problem has itself a long history, having been studied in [11], and more recently in [1] and [4]. For further information, see [8] for example. 
Lemma 2.1. Under hypothesis (11), the extremal problem given by (2) has at least one solution and every extremal vector $x_{0}$ saturates the constraint, in the sense that $\left\|B x_{0}-t_{0}\right\|=\varepsilon$. If, in addition, $\mathcal{Y}$ and $\mathcal{Z}$ are strictly convex, then the vector $x_{0}$ is unique.

Proof. The fact that the solution exists and saturates the constraint follows from the reflexivity of $\mathcal{X}$ and the assumption that $A$ has dense range, as in the proof of Lemma 2.1 of [9]. The uniqueness of $x_{0}$ in the case where $\mathcal{X}$ is strictly convex is not difficult to check. Indeed, if $x_{0}, x_{0}^{\prime}$ are distinct points in $\mathcal{C}_{t_{0}}^{\varepsilon}$ with $\left\|A x_{0}-t_{1}\right\|=\beta$ and $\left\|A x_{0}^{\prime}-t_{1}\right\|=\beta$, then the vector $x=\left(x_{0}+x_{0}^{\prime}\right) / 2$ also lies in $\mathcal{C}_{t_{0}}^{\varepsilon}$ and the strict convexity of the norm implies that $\left\|A x-t_{1}\right\|<\beta$ unless $A x_{0}-t_{1}=A x_{0}^{\prime}-t_{1}$, i.e., $A\left(x_{0}-x_{0}^{\prime}\right)=0$. But in that case $B x_{0}-t_{0} \neq B x_{0}^{\prime}-t_{0}$ by coprimeness, and the strict convexity of the norm now implies that $\left\|B x-t_{0}\right\|<\varepsilon$, which is a contradiction to the saturation condition.

The solution to the constrained extremal problem is given by the following theorem.

Theorem 2.1. Let $x_{0}$ be a solution to (21). Then there exists a negative constant $\mu$ such that

$$
B^{*} \nu_{\mathcal{Z}}\left(B x_{0}-t_{0}\right)=\mu A^{*} \nu \mathcal{Y}\left(A x_{0}-t_{1}\right) .
$$

Conversely, if $x_{0}$ satisfies (3) with $\mu<0$, and $\varepsilon=\left\|B x_{0}-t_{0}\right\|$, then $x_{0}$ satisfies (2) .

Proof. Suppose that $x_{0}$ is a solution of (2). Define the following subset of $\mathcal{X}$ :

$$
\mathcal{R}_{t_{1}}^{\beta}=\left\{x \in \mathcal{X}:\left\|A x-t_{1}\right\| \leq \beta\right\} .
$$

Our assumptions imply that $\operatorname{int}_{\mathcal{C}_{0}}^{\varepsilon}$ and $i n t \mathcal{R}_{t_{1}}^{\beta}$ are open, disjoint, nonempty convex sets. Hence by the Hahn-Banach theorem [16, Thm. 3.4] there is a functional $\Lambda \in \mathcal{X}^{*} \backslash\{0\}$ and a real number $\gamma$, such that

$$
\operatorname{Re} \Lambda(x)<\gamma<\operatorname{Re} \Lambda(y) \quad \text { for all } x \in \operatorname{int} \mathcal{C}_{t_{0}}^{\varepsilon}, y \in \operatorname{int} \mathcal{R}_{t_{1}}^{\beta} .
$$

Now $x_{0} \in \partial \mathcal{C}_{t_{0}}^{\varepsilon} \cap \partial \mathcal{R}_{t_{1}}^{\beta}$, and so $\operatorname{Re} \Lambda\left(x_{0}\right)=\gamma$.

Consider the following three hyperplanes in $\mathcal{X}$ :

$$
\begin{aligned}
\mathcal{H}_{1} & =\left\{x \in \mathcal{X}: \operatorname{Re} \nu\left(B x_{0}-t_{0}\right)\left(B x-t_{0}\right)=\varepsilon\right\}, \\
\mathcal{H}_{2} & =\left\{x \in \mathcal{X}: \operatorname{Re} \nu\left(A x_{0}-t_{1}\right)\left(A x-t_{1}\right)=\beta\right\}, \\
\mathcal{H}_{3} & =\{x \in \mathcal{X}: \operatorname{Re} \Lambda x=\gamma\} .
\end{aligned}
$$

Note that $x_{0} \in \mathcal{H}_{1} \cap \mathcal{H}_{2} \cap \mathcal{H}_{3}$. We want to prove that these hyperplanes coincide. To do this we prove that $\mathcal{H}_{1}=\mathcal{H}_{3}$; a similar argument shows that $\mathcal{H}_{2}=\mathcal{H}_{3}$.

So suppose that $\mathcal{H}_{1} \neq \mathcal{H}_{3}$. Then there is a point $z \in \mathcal{H}_{3} \backslash \mathcal{H}_{1}$, for otherwise $\mathcal{H}_{3} \subseteq \mathcal{H}_{1}$, and since both have real codimension 1 they must be equal. We may assume that

$$
\operatorname{Re\nu }\left(B x_{0}-t_{0}\right)\left(B z-t_{0}\right)<\varepsilon \quad \text { and } \quad \operatorname{Re} \Lambda z=\gamma
$$

for if $\operatorname{Re} \nu\left(B x_{0}-t_{0}\right)\left(B z-t_{0}\right)>\varepsilon$, then we may replace $z$ by $z^{\prime}=2 x_{0}-z \in \mathcal{H}_{3}$, which satisfies

$\operatorname{Re} \nu\left(B x_{0}-t_{0}\right)\left(B z^{\prime}-t_{0}\right)=2 \operatorname{Re} \nu\left(B x_{0}-t_{0}\right)\left(B x_{0}-t_{0}\right)-\operatorname{Re} \nu\left(B x_{0}-t_{0}\right)\left(B z-t_{0}\right)<\varepsilon$.

We now recall the following result. 
Proposition 2.1 ([6, p. 183]). Let $\mathcal{X}$ be a smooth Banach space, and let $x \in \mathcal{X}$ satisfy $\|x\|=1$. Suppose that $\operatorname{Re} \nu(x) y<1$ for some $y \in \mathcal{X}$. Then there exists a $\lambda \in(0,1)$ such that $\|\lambda x+(1-\lambda) y\|<1$.

It follows from this result and (4) that there is a number $\lambda \in(0,1)$ such that

$$
\left\|\lambda\left(B x_{0}-t_{0}\right)+(1-\lambda)\left(B z-t_{0}\right)\right\|<\varepsilon,
$$

and so

$$
\left\|B\left(\lambda x_{0}+(1-\lambda) z\right)-t_{0}\right\|<\varepsilon
$$

Let $x_{1}=\lambda x_{0}+(1-\lambda) z$. Then $x_{1} \in$ int $\mathcal{C}_{t_{0}}^{\varepsilon}$ and

$$
\begin{aligned}
\operatorname{Re} \Lambda\left(x_{1}\right) & =\lambda \operatorname{Re} \Lambda\left(x_{0}\right)+(1-\lambda) \operatorname{Re} \Lambda(z) \\
& =\lambda \gamma+(1-\lambda) \gamma=\gamma,
\end{aligned}
$$

which is a contradiction since $\operatorname{Re} \Lambda(x)<\gamma$ for all $x \in \operatorname{int} \mathcal{C}_{t_{0}}^{\varepsilon}$.

Given that $\mathcal{H}_{1}=\mathcal{H}_{2}=\mathcal{H}_{3}$, we have constants $\epsilon^{\prime}$ and $\beta^{\prime}$ such that the equations of $\mathcal{H}_{1}$ and $\mathcal{H}_{2}$ are, respectively,

$$
\operatorname{Re} B^{*} \nu\left(B x_{0}-t_{0}\right) x=\varepsilon^{\prime} \quad \text { and } \quad \operatorname{Re} A^{*} \nu\left(A x_{0}-t_{1}\right) x=\beta^{\prime} .
$$

Hence there is a nonzero constant $\mu$ such that

$$
\operatorname{Re} B^{*} \nu\left(B x_{0}-t_{0}\right)=\mu \operatorname{Re} A^{*} \nu\left(A x_{0}-t_{1}\right),
$$

and thus $B^{*} \nu\left(B x_{0}-t_{0}\right)=\mu A^{*} \nu\left(A x_{0}-t_{1}\right)$, since both functionals are complexlinear. It remains to show that $\mu<0$.

Suppose, to the contrary, that $\mu>0$. Since $A$ has dense range, there exists a $w_{0} \in \mathcal{X}$ such that $\left\|A w_{0}-t_{1}\right\|<\beta$. In particular,

$$
\begin{aligned}
\operatorname{Re} \nu\left(A x_{0}-t_{1}\right)\left(A w_{0}-t_{1}\right) & <\beta, \quad \text { and so } \\
\operatorname{Re} \nu\left(A x_{0}-t_{1}\right)\left(A x_{0}-A w_{0}\right) & >0,
\end{aligned}
$$

since $x_{0} \in \mathcal{H}_{2}$.

Now since $\mu>0$ we see from (5) that

$$
\begin{aligned}
\operatorname{Re} \nu\left(B x_{0}-t_{0}\right)\left(B x_{0}-B w_{0}\right) & >0, \quad \text { and so } \\
\operatorname{Re} \nu\left(B x_{0}-t_{0}\right)\left(B w_{0}-t_{0}\right) & <\operatorname{Re} \nu\left(B x_{0}-t_{0}\right)\left(B x_{0}-t_{0}\right) .
\end{aligned}
$$

Applying Proposition 2.1 once more, we see that there is a number $\eta \in(0,1)$ such that

$$
\begin{aligned}
\left\|\eta\left(B x_{0}-t_{0}\right)+(1-\eta)\left(B w_{0}-t_{0}\right)\right\| & <\varepsilon, \quad \text { i.e. }, \\
\left\|B\left(\eta x_{0}+(1-\eta) w_{0}\right)-t_{0}\right\| & <\varepsilon .
\end{aligned}
$$

Since $\left\|A w_{0}-t_{1}\right\|<\beta$ we have

$$
\left\|A\left(\eta x_{0}+(1-\eta) w_{0}\right)-t_{1}\right\| \leq \eta\left\|A x_{0}-t_{1}\right\|+(1-\eta)\left\|A w_{0}-t_{1}\right\|<\beta,
$$

which is a contradiction, since $\eta x_{0}+(1-\eta) w_{0} \in \mathcal{C}_{t_{0}}^{\varepsilon}$. This ends the proof of the first assertion of the theorem.

Conversely, suppose that $x_{0}$ satisfies (3) with $\mu<0$ and $\varepsilon=\left\|B x_{0}-t_{0}\right\|$. Let $x \in \mathcal{X}$ satisfy $\left\|B x-t_{0}\right\| \leq \varepsilon$. We have

$$
\operatorname{Re} \nu\left(B x_{0}-t_{0}\right)\left(B x-t_{0}\right) \leq \varepsilon=\operatorname{Re} \nu\left(B x_{0}-t_{0}\right)\left(B x_{0}-t_{0}\right),
$$

and then

$$
\begin{aligned}
\operatorname{Re} \nu\left(B x_{0}-t_{0}\right)\left(B\left(x-x_{0}\right)\right) & \leq 0, \quad \text { or, equivalently } \\
\operatorname{Re} B^{*} \nu\left(B x_{0}-t_{0}\right)\left(x-x_{0}\right) & \leq 0 .
\end{aligned}
$$


Using (3) we obtain

$$
\operatorname{Re} A^{*} \nu\left(A x_{0}-t_{1}\right)\left(x-x_{0}\right) \geq 0,
$$

which implies that

$$
\operatorname{Re} \nu\left(A x_{0}-t_{1}\right)\left(A x-t_{1}\right) \geq \operatorname{Re} \nu\left(A x_{0}-t_{1}\right)\left(A x_{0}-t_{1}\right)=\left\|A x_{0}-t_{1}\right\| .
$$

Hence $\left\|A x-t_{1}\right\| \geq\left\|A x_{0}-t_{1}\right\|$, and so the second assertion of the theorem is proved.

\section{Applications}

3.1. Bounded Completion Problem for Banach spaces. In this section we present the first application of the extremal problem analysed in Section 2, namely the so-called Bounded Completion Problem (BCP).

Let $\mathcal{E}$ be a Banach space, $\mathcal{M}$ a closed subspace of $\mathcal{E}$, and suppose that $\mathcal{E}$ can be written as a topological direct sum $\mathcal{E}=\mathcal{N}_{+} \oplus \mathcal{N}_{-}$of two closed subspaces. For $h \in \mathcal{N}_{-}$and $\varepsilon>0$, define

$$
\mathcal{C}_{h}^{\varepsilon}=\left\{g \in \mathcal{N}_{-}:\|g-h\| \leq \varepsilon\right\},
$$

which is clearly a closed convex nonempty set. Moreover, let $f \in \mathcal{N}_{+}$. We seek $g_{0} \in \mathcal{C}_{h}^{\varepsilon}$ such that

$$
\operatorname{dist}\left(f+g_{0}, \mathcal{M}\right)=\inf \left\{\operatorname{dist}(f+g, \mathcal{M}): g \in \mathcal{C}_{h}^{\varepsilon}\right\} .
$$

The original motivation of this problem was the special case $\mathcal{E}=L^{p}(\mathbb{T}), \mathcal{M}=$ $H^{p}(\mathbb{T})$, and $\mathcal{N}_{+}=L^{p}(I), \mathcal{N}_{-}=L^{p}(J)$ with $I$ and $J$ disjoint sets of positive Lebesgue measure such that $\mathbb{T}=I \cup J$. This was studied in the context of systems identifications for $p=\infty$ in [5] and for $p=2$ in [12] (see also [8] for a survey of this and related problems).

We shall see that the case $1<p<\infty$ can be considered as a corollary of the following result. Let $\pi: \mathcal{E} \rightarrow \mathcal{E} / \mathcal{M}$ denote the quotient mapping.

We begin with two preliminary lemmas.

Lemma 3.1. If $\mathcal{E}$ is smooth and reflexive, then $\mathcal{E} / \mathcal{M}$ is smooth.

Proof. It follows from the hypotheses that $\mathcal{E}^{*}$ is strictly convex ([14], p. 481), and so its subspace $\mathcal{M}^{\perp}$ is strictly convex. But $\mathcal{M}^{\perp}$ is isometrically isomorphic to $(\mathcal{E} / \mathcal{M})^{*}$, and hence $\mathcal{E} / \mathcal{M}$ is smooth since its dual is strictly convex ([14], p. 481).

Denote by $P_{\mathcal{N}_{+}}$the continuous projection of $\mathcal{E}$ onto $\mathcal{N}_{+}$with kernel $\mathcal{N}_{-}$.

Lemma 3.2. The following conditions are equivalent:

(a) $P_{\mathcal{N}_{+}} \mathcal{M}$ is dense in $\mathcal{N}_{+}$;

(b) $\mathcal{M}^{\perp} \cap \mathcal{N}_{-}^{\perp}=\{0\}$;

(c) $\pi_{\mid \mathcal{N}_{-}}$has dense range.

Proof. The equivalence of (a) and (b) is established in [17, Lem. 1.1] by means of the Hahn-Banach theorem. It remains to prove that (b) is equivalent to (c). Condition (c) is equivalent to the injectivity of $\left(\pi_{\mid \mathcal{N}_{-}}\right)^{*}:(\mathcal{E} / \mathcal{M})^{*} \rightarrow \mathcal{N}_{-}^{*}$. Let $\Phi: \mathcal{M}^{\perp} \rightarrow(\mathcal{E} / \mathcal{M})^{*}$ denote the canonical isometric isomorphism. For $x \in \mathcal{N}_{-}$and $\Lambda \in \mathcal{M}^{\perp}$ we have

$$
\left(\pi_{\mid \mathcal{N}_{-}}\right)^{*} \Phi(\Lambda)(x)=\Phi(\Lambda)(x+\mathcal{M})=\Lambda(x) .
$$

So $\left(\pi_{\mid \mathcal{N}_{-}}\right)^{*}$ is injective if and only if whenever $\Lambda \in \mathcal{M}^{\perp}$ and $\Lambda(x)=0$ for all $x \in \mathcal{N}_{-}$, we have $\Lambda=0$. This is just condition (b). 
Theorem 3.1. Suppose that $\mathcal{E}$ is a reflexive, smooth and strictly convex Banach space, and that the hypotheses of Lemma 3.2 are satisfied. Then the Bounded Completion Problem has a unique solution $g_{0}$ satisfying

$$
\nu_{\mathcal{N}_{-}}\left(g_{0}-h\right)=\mu\left(\pi_{\mid \mathcal{N}_{-}}\right)^{*} \nu_{\mathcal{E} / \mathcal{M}}\left(\pi\left(g_{0}+f\right)\right)
$$

for some $\mu<0$ such that $\left\|g_{0}-h\right\|=\varepsilon$.

Proof. Take $\mathcal{X}=\mathcal{N}_{-}, \mathcal{Y}=\mathcal{E} / \mathcal{M}, \mathcal{Z}=\mathcal{N}_{-}, A=\pi_{\mid \mathcal{N}_{-}}, B=I d$ on $\mathcal{N}_{-}, t_{0}=h$ and $t_{1}=-\pi f$. Then we immediately see that $\mathcal{X}$ is reflexive, smooth and strictly convex, $\mathcal{Z}$ is smooth, and $A$ and $B$ are coprime. By Lemma $3.1 \mathcal{Y}$ is smooth and by Lemma $3.2 A$ has dense range. Then by Theorem 2.1, the result now follows (with $g_{0}=x_{0}$ ).

This translates into a more explicit condition in the case $\mathcal{E}=L^{p}(\mathbb{T})$ described above, since, identifying $L^{p}(\mathbb{T})^{*}$ with $L^{q}(\mathbb{T})$, where $q=p /(p-1)$, we have

$$
\nu_{\mathcal{N}_{-}}\left(g_{0}-h\right)=\left(\overline{g_{0}-h}\right)\left|g_{0}-h\right|^{p-2}\left\|g_{0}-h\right\|^{1-p} .
$$

3.2. Construction of hyperinvariant subspaces. We now present the second application of the extremal problem analysed in Section 2 .

From now on, let $\mathcal{X}$ denote a reflexive, smooth complex Banach space. (Note that, indeed, any reflexive Banach space can be given an equivalent smooth norm by [10, p. 289], so that in searching for hyperinvariant subspaces we shall not lose any additional generality by imposing the smoothness condition.) Suppose that $T \in \mathcal{L}(\mathcal{X})$ has dense range, that $\varepsilon>0$, that $t_{0} \in \mathcal{X}$ with $\left\|t_{0}\right\|>\varepsilon$, and that $n$ is a positive integer. Consider $\mathcal{C}_{n, t_{0}}^{\varepsilon}$ the subset of $\mathcal{X}$ defined by

$$
\mathcal{C}_{n, t_{0}}^{\varepsilon}=\left\{x \in \mathcal{X}:\left\|T^{n} x-t_{0}\right\| \leq \varepsilon\right\} .
$$

Since $T^{n}$ has dense range, $\mathcal{C}_{n, t_{0}}^{\varepsilon}$ is a nonempty closed convex subset of $\mathcal{X}$. A backward minimal vector for $T^{n}$ is a vector $x_{n, t_{0}}^{\varepsilon} \in \mathcal{C}_{n, t_{0}}^{\varepsilon}$ satisfying

$$
\left\|x_{n, t_{0}}^{\varepsilon}\right\|=\inf \left\{\|x\|: x \in \mathcal{C}_{n, t_{0}}^{\varepsilon}\right\} .
$$

Note that the condition $\left\|t_{0}\right\|>\varepsilon$ implies that $0 \notin \mathcal{C}_{n, t_{0}}^{\varepsilon}$ and thus $x_{n, t_{0}}^{\varepsilon} \neq 0$.

Proposition 3.1. In the above situation, an optimal $x_{n}=x_{n, t_{0}}^{\varepsilon}$ exists and satisfies $\left\|T^{n} x_{n}-t_{0}\right\|=\varepsilon$ and $T^{* n} \nu\left(T^{n} x_{n}-t_{0}\right)=\mu_{n} \nu\left(x_{n}\right)$ for some $\mu_{n}<0$. Thus if $z \in \mathcal{X}$ we have

$$
\nu\left(x_{n}\right) z=0 \quad \Longleftrightarrow \quad \nu\left(T^{n} x_{n}-t_{0}\right)\left(T^{n} z\right)=0 .
$$

Further, if $\mathcal{X}$ is strictly convex, then the backward minimal vector $x_{n, t_{0}}^{\varepsilon}$ is unique.

Proof. The existence of $x_{n}=x_{n, t_{0}}^{\varepsilon}$ and its two basic properties follows directly from Theorem 2.1, on taking $\mathcal{Y}=\mathcal{Z}=\mathcal{X}, A=I d, B=T^{n}$ and $t_{1}=0$. Next,

$$
\begin{aligned}
\nu\left(x_{n}\right) z & =\mu_{n}^{-1} T^{* n} \nu\left(T^{n} x_{n}-t_{0}\right) z \\
& =\mu_{n}^{-1} \nu\left(T^{n} x_{n}-t_{0}\right)\left(T^{n} z\right),
\end{aligned}
$$

which gives (6). Finally the uniqueness of $x_{n}$ in the case that $\mathcal{X}$ is strictly convex follows from Lemma 2.1 . 
Theorem 3.2. Let $\mathcal{X}$ be a reflexive smooth Banach space, and $T \in \mathcal{L}(\mathcal{X})$ an operator with dense range. Suppose that there exist $\varepsilon>0$ and $t_{0} \in \mathcal{X}$ such that $\left\|t_{0}\right\|>\varepsilon$ for which there exists a subsequence $\left(n_{k}\right)_{k \geq 1}$ of $\mathbb{N}$ satisfying

$$
\lim _{k \rightarrow \infty} \frac{\left\|x_{n_{k}-1}\right\|}{\left\|x_{n_{k}}\right\|}=0
$$

and $\left(T^{n_{k}} x_{n_{k}-1}\right)_{k \geq 1}$ converges in norm to a nonzero vector. Then $T$ has a nontrivial hyperinvariant subspace.

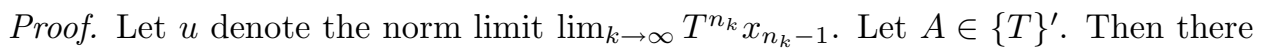
exist $\alpha_{n_{k}} \in \mathbb{C}$ such that

$$
A x_{n_{k}-1}=\alpha_{n_{k}} x_{n_{k}}+w_{n_{k}}, \quad \text { where } \quad \nu\left(x_{n_{k}}\right) w_{n_{k}}=0 .
$$

Therefore

$$
\operatorname{Re} \nu\left(x_{n_{k}}\right)\left(A x_{n_{k}-1}\right)=\alpha_{n_{k}}\left\|x_{n_{k}}\right\|
$$

and so

$$
\left|\alpha_{n_{k}}\right|=\frac{\left|\operatorname{Re\nu }\left(x_{n_{k}}\right)\left(A x_{n_{k}-1}\right)\right|}{\left\|x_{n_{k}}\right\|} \leq\|A\| \frac{\left\|x_{n_{k}-1}\right\|}{\left\|x_{n_{k}}\right\|} \rightarrow 0
$$

by assumption.

Now since $A T=T A$ we have $A T^{n_{k}} x_{n_{k}-1}=\alpha_{n_{k}} T^{n_{k}} x_{n_{k}}+T^{n_{k}} w_{n_{k}}$, and so $\nu\left(T^{n_{k}} x_{n_{k}}-t_{0}\right)\left(A T^{n_{k}} x_{n_{k}-1}\right)=\alpha_{n_{k}} \nu\left(T^{n_{k}} x_{n_{k}}-t_{0}\right) T^{n_{k}} x_{n_{k}}+\nu\left(T^{n_{k}} x_{n_{k}}-t_{0}\right) T^{n_{k}} w_{n_{k}}$, and the second term is zero by (6). Hence

$$
\nu\left(T^{n_{k}} x_{n_{k}}-t_{0}\right)\left(A T^{n_{k}} x_{n_{k}-1}\right)=\alpha_{n_{k}} \nu\left(T^{n_{k}} x_{n_{k}}-t_{0}\right) T^{n_{k}} x_{n_{k}} \rightarrow 0,
$$

since $\alpha_{n_{k}} \rightarrow 0$ and $\left(T^{n_{k}} x_{n_{k}}\right)_{k \geq 1}$ is bounded. We conclude that

$$
\nu\left(T^{n_{k}} x_{n_{k}}-t_{0}\right)(A u) \rightarrow 0 .
$$

By passing to a subsequence and by relabelling, we may suppose that $\left(\nu\left(T^{n_{k}} x_{n_{k}}-t_{0}\right)\right)_{k \geq 1}$ converges weakly to a functional $\Phi \in \mathcal{X}^{*}$. Thus $\Phi(A u)=0$. Now

$$
\operatorname{Re} \nu\left(T^{n_{k}} x_{n_{k}}-t_{0}\right)\left(T^{n_{k}} x_{n_{k}}\right)=\mu_{n_{k}} \operatorname{Re} \nu\left(x_{n_{k}}\right) x_{n_{k}}=\mu_{n_{k}}\left\|x_{n_{k}}\right\|<0
$$

and

$$
\operatorname{Re} \nu\left(T^{n_{k}} x_{n_{k}}-t_{0}\right)\left(T^{n_{k}} x_{n_{k}}-t_{0}\right)=\left\|T^{n_{k}} x_{n_{k}}-t_{0}\right\|=\varepsilon .
$$

Subtracting (91) from (8) we conclude that

$$
\operatorname{Re} \nu\left(T^{n_{k}} x_{n_{k}}-t_{0}\right) t_{0}=-\varepsilon+\mu_{n_{k}}\left\|x_{n_{k}}\right\|<-\varepsilon \text {. }
$$

Hence $\operatorname{Re} \Phi\left(t_{0}\right) \leq-\varepsilon$, and so $\Phi \neq 0$. Moreover, $\Phi(A u)=0$ for all $A \in\{T\}^{\prime}$.

Now let $\mathcal{M}=\operatorname{span}\left\{A u: A \in\{T\}^{\prime}\right\}$, which is a closed hyperinvariant subspace for $T$. Now $u \neq 0$, by hypothesis, so $\mathcal{M} \neq\{0\}$ and also $\mathcal{M} \neq \mathcal{X}$, since $\Phi(v)=0$ for all $v \in \mathcal{M}$.

Recall Lemma 1 in [3].

Lemma 3.3. Let $T \in \mathcal{L}(\mathcal{X})$ with dense range, $\varepsilon>0$, and $t_{0} \in \mathcal{X}$ such that $\left\|t_{0}\right\|>\varepsilon$. Denote by $\left(x_{n}\right)_{n \geq 1}$ the sequence of backward minimal vectors. If $T$ is quasinilpotent, then there exists a subsequence $\left(x_{n_{k}}\right)_{k \geq 1}$ of $\left(x_{n}\right)_{n \geq 1}$ such that

$$
\lim _{k \rightarrow \infty} \frac{\left\|x_{n_{k}-1}\right\|}{\left\|x_{n_{k}}\right\|}=0
$$


In conjunction with the above theorem, we obtain the following result which provides an extension of the results of [3] and [9].

Corollary 3.1. Let $\mathcal{X}$ be a reflexive Banach space, and let $T \in \mathcal{L}(\mathcal{X})$ be a quasinilpotent operator with dense range. Suppose that there exist $\varepsilon>0, t_{0} \in \mathcal{X}$ such that $\left\|t_{0}\right\|>\varepsilon$ and $\left(T^{n} x_{n}\right)_{n \geq 1}$ converges in norm. Then $T$ has a nontrivial hyperinvariant subspace.

Proof. Without loss of generality, we may suppose that $T$ is injective. Also $\left\|T^{n} x_{n}\right\|$ $\geq\left\|t_{0}\right\|-\varepsilon$, which easily implies that $\lim _{n \rightarrow \infty} T^{n} x_{n-1}$ exists and is nonzero. The result now follows from Theorem 3.2 .

Now we can deduce a well-known result due to Lomonosov [13]. See for example the accounts in [15] and [7].

Corollary 3.2. Let $\mathcal{X}$ be a reflexive Banach space and let $K \in \mathcal{L}(\mathcal{X})$ be a nonzero compact operator. Then $K$ has a nontrivial hyperinvariant subspace.

Proof. This follows easily by noting that we may without loss of generality assume that $K$ is quasinilpotent and injective with dense range, and hence the first hypothesis of Theorem 3.2 is satisfied; the second hypothesis is an immediate consequence of compactness.

The following examples, which are closely related to a Hilbert space example presented in [9], involve operators that are neither compact nor quasinilpotent.

Example 3.1. Take $1<p<\infty$ and define $T: \ell^{p}(\mathbb{Z}) \rightarrow \ell^{p}(\mathbb{Z})$ by $T e_{n}=\lambda_{n} e_{n+1}$, where $\left(e_{n}\right)_{n \in \mathbb{Z}}$ is the standard basis of $\ell^{p}(\mathbb{Z})$ and $\left(\lambda_{n}\right)_{n \in \mathbb{Z}}$ is a bounded complex sequence with $\lambda_{n} \neq 0$ for all $n$. Then $T^{*}: \ell^{q}(\mathbb{Z}) \rightarrow \ell^{q}(\mathbb{Z})$ satisfies $T^{*} e_{n}=\lambda_{n-1} e_{n-1}$ for all $n$. Clearly

$$
\left\|T^{n}\right\|=\sup \left\{\left|\lambda_{k} \cdots \lambda_{k+n-1}\right|: k \in \mathbb{Z}\right\} .
$$

Using the notation above, let us take $t_{0}=e_{r}$ for some $r \in \mathbb{Z}$ and $0<\varepsilon<1$; it is then easily verified that $T^{n} x_{n}=(1-\varepsilon) e_{r}$ and

$$
x_{n}=\frac{1-\varepsilon}{\lambda_{r-1} \cdots \lambda_{r-n}} e_{r-n} .
$$

Thus $T^{n} x_{n-1}=\lambda_{r}(1-\varepsilon) e_{r+1}$, so provided that $\inf _{j<0}\left|\lambda_{j}\right|=0$, the hypotheses of Theorem 3.2 are satisfied, and $T$ has a nontrivial hyperinvariant subspace, namely $\mathcal{M}=\operatorname{span}\left\{A e_{r+1}: A \in\{T\}^{\prime}\right\}$.

A similar argument applied to the adjoint shows that $T^{*}$, and hence $T$, has a nontrivial hyperinvariant subspace if $\inf _{j \geq 0}\left|\lambda_{j}\right|=0$. Thus if $\inf _{j \in \mathbb{Z}}\left|\lambda_{j}\right|=0$, i.e., if $T$ is noninvertible, then $T$ always has a nontrivial hyperinvariant subspace. We are grateful to Dr. S.P. Eveson for this observation.

It is not difficult to write down sequences $\left(\lambda_{n}\right)_{n \in \mathbb{Z}}$ for which $T$ is neither quasinilpotent nor compact (see, for example, (10) below).

An analogous construction can be performed in various other Banach spaces with 1-unconditional bases; we omit the details.

Example 3.2. Another example, which, in the case $p=2$ coincides with the previous one, can be obtained by taking $\mathcal{X}=L^{p}(\mathbb{T})$, and defining $e_{n} \in \mathcal{X}$ by $e_{n}(z)=z^{n}$ for $n \in \mathbb{Z}$ and $z \in \mathbb{T}$. We may define $T: L^{p}(\mathbb{T}) \rightarrow L^{p}(\mathbb{T})$ by $T e_{n}=$ $\lambda_{n} e_{n+1}$, for each $n$, where $\left(\lambda_{n}\right)_{n \in \mathbb{Z}}$ is a sequence of nonzero complex numbers such 
that this operator has a bounded extension to $L^{p}(\mathbb{T}$ ) (clearly it will have dense range). For example, the sequence

$$
\lambda_{n}= \begin{cases}-1 / n & \text { for } n<0 \\ 1 & \text { for } n \geq 0\end{cases}
$$

is a suitable choice, and in this case the operator $T$ is neither quasinilpotent nor compact.

Let us take $t_{0}=e_{r}$ for a fixed $r \in \mathbb{Z}$ and take $0<\varepsilon<1$. We claim that for every $n$ we have $x_{n}=a_{n} e_{r-n}$ for some constant $a_{n}$. To see this we apply the converse to Theorem 2.1 noting that $T^{* n} \nu\left(T^{n} x_{n}-t_{0}\right)=\mu_{n} \nu\left(x_{n}\right)$ for all $n$, because $\nu\left(e_{s}\right)=e_{-s}$ for every $s$. Note that $\mu_{n}<0$, and then the choice of $a_{n}$ such that $a_{n} \lambda_{r-n} \cdots \lambda_{r-1}=1-\varepsilon$ ensures that $\left\|T^{n} x_{n}-t_{0}\right\|=\varepsilon$ and $T^{n} x_{n-1}=$ $\lambda_{r}(1-\varepsilon) e_{r+1}$. Thus the hypotheses of Theorem 3.2 are satisfied and $T$ has a nontrivial hyperinvariant subspace.

\section{ACKNOWLEDGEMENTS}

The authors are grateful to the Franco-British Alliance programme and the European Research Training Network in Analysis and Operators for financial support. They also thank Dr. D.L. Salinger for useful discussions.

\section{REFERENCES}

1. D. Alpay, L. Baratchart, and J. Leblond. Some extremal problems linked with identification from partial frequency data. In R. F. Curtain, A. Bensoussan, and J.-L. Lions, editors, Analysis and optimization of systems: state and frequency domain approaches for infinitedimensional systems (Sophia-Antipolis, 1992), pages 563-573. Springer-Verlag, Berlin, 1993. MR 93i:93005

2. G. Androulakis. A note on the method of minimal vectors. Trends in Banach spaces and operator theory, Contemporary Mathematics, Ed. A. Kaminska, to appear.

3. S. Ansari and P. Enflo. Extremal vectors and invariant subspaces. Trans. Amer. Math. Soc., 350:539-558, 1998. MR 98d:47019

4. L. Baratchart and J. Leblond. Hardy approximation to $L^{p}$ functions on subsets of the circle with $1 \leq p<\infty$. Constr. Approx., 14(1):41-56, 1998. MR 98i:41041

5. L. Baratchart, J. Leblond, and J. R. Partington. Problems of Adamjan-Arov-Krein type on subsets of the circle and minimal norm extensions. Constr. Approx., 16(3):333-357, 2000. MR 2001j:41020

6. B. Beauzamy. Introduction to Banach spaces and their geometry. North-Holland Publishing Co., Amsterdam, 1982. Notas de Matemática [Mathematical Notes], 86. MR 84g:46017

7. I. Chalendar and J. Esterle. Le problème du sous-espace invariant. In J.-P. Pier, editor, Development of mathematics 1950-2000, pages 235-267. Birkhäuser, Basel, 2000. MR 2001i:47005

8. I. Chalendar, J. Leblond, and J. R. Partington. Approximation problems in some holomorphic spaces, with applications. In A. A. Borichev and N. K. Nikolski, editors, Systems, Approximation, Singular Integral Operators, and Related Topics, Proceedings of IWOTA 2000, pages 143-168. Birkhäuser, 2001. MR 2002k:00031

9. I. Chalendar and J. R. Partington. Constrained approximation and invariant subspaces. J. Math. Anal. Appl., 280:176-187, 2003.

10. R. Deville, G. Godefroy, and V. Zizler. Smoothness and renormings in Banach spaces. Longman Scientific \& Technical, Harlow, 1993. MR 94d:46012

11. M. G. Kreĭn and P. Ja. Nudel'man. Approximation of functions in $L_{2}\left(\omega_{1}, \omega_{2}\right)$ by transmission functions of linear systems with minimal energy. Problemy Peredači Informacii, 11(2):37-60, 1975. English translation. MR 58:1947

12. J. Leblond and J. R. Partington. Constrained approximation and interpolation in Hilbert function spaces. J. Math. Anal. Appl., 234(2):500-513, 1999. MR 2000e:41033 
13. V. I. Lomonosov. Invariant subspaces for operators commuting with compact operators. Funct. Anal. Appl., 7:213-214, 1973.

14. R. E. Megginson. An introduction to Banach space theory. Springer-Verlag, New York, 1998. MR 99k:46002

15. H. Radjavi and P. Rosenthal. Invariant subspaces. Springer-Verlag, New York, 1973. MR 51:3924

16. W. Rudin. Functional analysis. McGraw-Hill, Inc., New York, second edition, 1991. MR 92k:46001

17. M. Smith. Constrained approximation in Banach spaces. Constr. Approx., to appear.

18. V. G. Troitsky. Minimal vectors in arbitrary Banach spaces. Preprint, 2002.

Institut Girard Desargues, UfR de Mathématiques, Université Claude Bernard Lyon 1, 69622 Villeurbanne Cedex, France

E-mail address: chalenda@igd.univ-lyon1.fr

School of Mathematics, University of Leeds, Leeds LS2 9JT, United Kingdom

E-mail address: J.R.Partington@leeds.ac.uk

Department of Mathematics, University of York, Heslington, York, YO10 5DD, UNITED KINGDOM

E-mail address: mps6@york.ac.uk 